OPEN ACCESS

Edited by:

Jian Gao,

Second Affiliated Hospital of Dalian Medical University, China

Reviewed by: Hong Qi,

Peking University Third Hospital, China Xiuming Jin,

Zhejiang University, China

${ }^{*}$ Correspondence:

Lijun Zhang

lijunzhangw@gmail.com Xun Wang

wangxun1980@126.com

tThese authors have contributed equally to this work

Specialty section: This article was submitted to Inflammation Pharmacology,

a section of the journal

Frontiers in Pharmacology

Received: 29 June 2021

Accepted: 26 July 2021

Published: 05 August 2021

Citation:

Yu L, Yu C, Dong H, Mu Y, Zhang R, Zhang $Q$, Liang W, Li W, Wang $X$ and

Zhang $L$ (2021) Recent Developments

About the Pathogenesis of Dry Eye

Disease: Based on Immune

Inflammatory Mechanisms.

Front. Pharmacol. 12:732887.

doi: 10.3389/fphar.2021.732887

\section{Recent Developments About the Pathogenesis of Dry Eye Disease: Based on Immune Inflammatory Mechanisms}

\author{
Lifei $\mathrm{Yu}^{1 \dagger}$, Chunjing $\mathrm{Yu}^{1 \dagger}$, He Dong ${ }^{1 \dagger}$, Yanan Mu ${ }^{1}$, Rui Zhang ${ }^{1}$, Qiaosi Zhang ${ }^{1}$, Wei Liang ${ }^{1}$, \\ Wenjia $\mathrm{Li}^{1}$, Xun Wang ${ }^{2 *}$ and Lijun Zhang ${ }^{1 *}$ \\ ${ }^{1}$ Department of Ophthalmology, The Third People's Hospital of Dalian, Non-Directly Affiliated Hospital of Dalian Medical \\ University, Dalian, China, ${ }^{2}$ Department of Neurosurgery, The Third People's Hospital of Dalian, Non-Directly Affiliated Hospital of \\ Dalian Medical University, Dalian, China
}

Dry eye disease is a common and frequently occurring ophthalmology with complex and diverse causes, and its incidence is on the upward trend. The pathogenesis of DED is still completely clear. However, the immune response based on inflammation has been recognized as the core basis of this disease. In this review, we will systematically review the previous research on the treatment of DED in immune inflammation, analyze the latest views and research hotspots, and provide reference for the prevention and treatment of DED.

Keywords: Dry eye disease, immunity, inflammation, pharmaceuticals, treatment

\section{INTRODUCTION}

Dry eye disease (DED) is newly defined as eye surface disease caused by a variety of factors, tear film instability, increased osmotic pressure, ocular surface inflammation and damage, and neurosensory abnormalities play a pathogenic role, characterized by the loss of tear film balance accompanied by eye symptoms. These include dryness, foreign body sensation, burning sensation, itching sensation, photophobia, red eyes, blurred vision, fluctuating vision, and visual fatigue. In severe cases, corneal epithelial exfoliation, filamentous adhesion, and conjunctival lesions may occur (Stapleton et al., 2017). The global prevalence of dry eye disease is $5-50 \%$, while the incidence in China is 45\%, which is a high incidence area (Guo et al., 2010; Farrand et al., 2017; Stapleton et al., 2017). With the popularity of electronic products, makeup, contact lenses, environmental pollution and other influences, the number of patients with dry eye disease will continue to rise at a rate of more than $10 \%$ per year, and tend to be younger. The pathogenesis of dry eye has not yet been fully elucidated, but the eye surface immune inflammatory response as the focus of the mechanism has been increasingly concerned. In the classification of etiology, Dry Eye Workshop II regards the imbalance of tear film homeostasis as the main feature of dry eye and the core of pathophysiology, whether it is water-based tear deficiency type or over-evaporation type (Nelson et al., 2017). This process is caused by the increase of Th17 and chemokines in the ocular surface of DED patients, which breaks the normal ocular surface immune balance and leads to the immune homeostasis in the tear membrane (Kodati et al., 2014). In the clinical treatment of DED, anti-inflammatory drugs represented by cyclosporine A and immunomodulatory drugs represented by lifitegrast can play a good ameliorative effect (Wan et al., 2015; Perez et al., 2016). Therefore, it is of strategic significance to further study the immune inflammatory mechanism of DED. 


\section{IMMUNE RESPONSE AND DED}

The ocular surface immune response is a rigorous and complex regulatory process designed to protect and defend the ocular surface, but if the regulation is maladjusted, it will lead to DED (Pflugfelder and Paiva, 2017). Immune response can be divided into innate immunity and adaptive immunity. The inherent response is the innate immunity of the human body, known as the first natural defense line, which mainly includes macrophages, monocytes, dendritic cells, neutrophils and natural killer cells, etc. Adaptive immunity is acquired immunity, which generally forms a highly targeted immune process after the invasion of certain pathogenic microorganisms. The two immune modes are jointly involved in the immune regulation of dry eye disease (Schaumburg et al., 2011).

\section{Innate Immune Response of Ocular Surface}

In the innate immunity, the human body has formed a natural physical barrier composed of sugar calyx, conjunctival epithelium, mucin, cornea, and a series of antimicrobial defense proteins in tears in the long time of survival and evolution. This barrier is a necessary part to ensure the relative safety of the eyes exposed to the external environment. After the occurrence of dry eye, the high osmotic state of patients can prevent the defense system from taking effect, and further aggravate the immune inflammatory response by directly activating the MAPK pathway to activate interleukin and tumor necrosis factor (Milner et al., 2017). At the same time, in the process of immune inflammation, Toll-like receptor signal transduction will lead to the activation of immune cells, further aggravating the inflammatory response (Lee et al., 2012).

Inflammatory immune response IIR is the most important type of innate immune response, among which macrophage and dendritic cells (DC) are common inflammatory immune cells, among which macrophages are divided into M1 and M2 cells. The former is related to cellular response, while the latter plays a regulatory role. DC are divided into myeloid cells (DC1) and lymphoid cells (DC2). In dry eye disease, DC2 cells are mainly involved in immune regulation, and the two types of cells combine with the expression of various factors, such as interleukin and tumor necrosis factor, leading to the continuous increase of inflammatory receptor levels leading to dry eye disease (Jaafar et al., 2009).

\section{Adaptive Immune Response of Ocular Surface}

The abundant presence of $\mathrm{CD}^{+}{ }^{+} \mathrm{T}$ cells in the adaptive immune response and cyclosporine in the treatment of DED suggest that adaptive immunity also plays an important role in DED. During the adaptive immune phase, the production of antigen-specific $\mathrm{T}$ cells in regional lymph nodes induces migration to the ocular surface in response to ocular stress. In this stage, the proliferation and amplification of $\mathrm{T}$ cells in the ocular surface cause injury, restart the acute proinflammatory innate response, and with the loss of immune regulation, trigger a vicious cycle of pathological immune response (Baudouin, 2001). DC is the most powerful known APC that can activate initial T cells, and play a dual role in the initiation and regulation of immune response, connecting innate immunity and adaptive immunity (Hackstein et al., 2016; Pan et al., 2016). In diabetes-associated dry eyes, advanced glycosylation end products can directly promote the maturation of DC and induce specific immune response of $\mathrm{CD} 4^{+} \mathrm{T}$ cells, and the increased number and abnormal function of DC can induce adaptive immune response. (Charles et al., 2009; Surenda et al., 2011). Inflammatory factors in patients with DED can be amplified by DC, for example by upregulating the expression of TLR7 and activating the secretion of IFN- $\gamma$ (Hui et al., 2010). IFN- $\gamma$ induces the transformation of $\mathrm{B}$ cells into $\mathrm{DC}$ cells to produce antigen-specific antibodies, which are further activated by CD40 signaling molecules to produce large quantities of IL-6. IL-6 can promote the differentiation of Th17 cells by enhancing and inducing transcriptional activators, and secrete proinflammatory cytokine IL-17 to enhance immune response (Jego et al., 2003). The IL-4 secreted by mature PDC stimulates the differentiation of $\mathrm{CD}^{+} \mathrm{T}$ cells into $\mathrm{Th} 2$ cells, which can secrete a large number of inhibitory cytokines including IL-4, IL-10, and IL-13 upon activation. IL-4 increases $B$ cell infiltration in lacrimal glands, thereby promoting the production of autoantibodies against acinar epithelial cells and contributing to the pathogenesis of DED (Abehsira-Amar et al., 1992).

\section{IMMUNE-BASED INFLAMMATION MECHANISMS IN DED}

Inflammation is the most common and important risk factor for DED, and studies have shown that patients with DED can detect large amounts of lymphocyte infiltration in the ocular surface and lacrimal gland tissue, at the same time, the secretion of lactoferrin decreased, the cell inflammatory factors, leading to further expansion of the scope of inflammation. Ophthalmic surface inflammation is both an initial cause and a subsequent consequence of DED (Baudouin, 2001). When the body is exposed to external stimuli or homeostasis disorders, the initiation and elimination of ocular surface inflammation are usually controlled by immunomodulatory processes. The continued inflammatory response amplifies the immune response, especially the adaptive response. This process will increase the activity of mAPC, as well as the production and recruitment of $\mathrm{CD}^{+} \mathrm{Th}$ cells in the ocular surface. When immune regulation is insufficient to eliminate inflammation or is bypassed, the activity of effector $\mathrm{T}$ cells will be immediately disregulated, mainly manifested by increased release of proinflammatory cytokines, which will lead to further inflammation and injury. The new inflammation and damage then restarts the innate immune response, creating a vicious cycle (Stern et al., 2010; Stern et al., 2013; Periman et al., 2020). At the molecular level, studies on the relationship between dry eye and inflammation mainly focus on cytokines, chemokines and signal transduction pathways. 
Throughout the inflammatory response, immune cells release pro-inflammatory cytokines and chemokines, recruiting more immune cells and ultimately leading to a vicious cycle of inflammation. Current studies on inflammatory mediators associated with DED include IL-1 $\alpha$, IL-1 $\beta$, IL-1R, IL-2, IL-4, IL-6, IL-8, IL-10, IL-12, IL-13, IL-17, IL-23, CCL-2, CCL-3, CCL5, CCL-19, CCL-20, CCL-21, CCR2, CCR6, CCR7, CX3CL1, CXCL9, CXCL10, CXCL12, CXCR3, CXCR4, EGF, ICAM-1, IFN- $\gamma$, MMP-1, MMP-3, MMP-9, MMP-13, TNF- $\alpha$, TGF- $\beta 2$, VCAM-1, and NF-кB (Matsuda et al., 1998; Matsuda and Koyasu, 2000; Marsland et al., 2005; Chauhan et al., 2009; Du et al., 2009; Lam et al., 2009; Gollmer et al., 2009; Enríquez-de-Salamanca et al., 2010; Chen et al., 2011; Schaumburg, et al., 2011; Zhang et al., 2011; Zhang et al., 2012; Coursey et al., 2013; Dohlman et al., 2013; Li et al., 2013; Marko et al., 2013; Barbosa et al., 2014; Duque and Descoteaux, 2014; Ji et al., 2014; Kodati et al., 2014; Mesraoua et al., 2014; Zhang et al., 2014; Ames and Galor, 2015; Corrales et al., 2015; Contreras-Ruiz and Masli. 2015; Pierre et al., 2018; Seung et al., 2019).

IL-32 and IL-33 are new family of IL-1 cytokines involved in a variety of inflammatory diseases. A new study has found that IL-32 and IL-32-induced TSLP is a key cytokine involved in the inflammatory response through the corneal epithelial caspase-1 and NF- $\mathrm{kB}$ signaling pathways, providing a new molecular target for ocular surface inflammatory diseases (Jing et al., 2018). Another study found elevated IL-33 mRNA and protein levels in HCONEC cells under hypertonic conditions (Wang and Zhang, 2019). IL-33 and its receptor ST2 protein levels were higher in CIC of DE patients and correlated with the severity of the disease. In addition, the activated type 2 helper $\mathrm{T}$ (Th2) cells in the tears of $\mathrm{DE}$ patients released increased concentrations of IL-13 and IL-5, and the IL-33/ST2 pathway may play a role in the initiation of ophthalmic surface inflammation regulation. IL-33 mRNA and protein levels were increased in the corneal tissues of mice and human corneal epithelial cells (HCECs) infected with Aspergillus fumigatus. IL-33 also promoted the proliferation of HCECs cells through its receptor ST2. In addition, IL-33/ST2/p38 signaling pathway plays an important role in enhancing the inflammatory response of HCECs to Aspergillus fumigatus infection (You et al., 2019).

\section{Immunoregulatory Molecules and Inflammation}

Corneal epithelial cells (CECs) are the main target tissues for the immunomodulatory response of DED. More and more studies have been conducted on the expression of immunomodulatory molecules in corneal epithelial cells.

Pigment epithelium-derived factor (PEDF) is a $50 \mathrm{kDa}$ secreted glycoprotein with well-established anti-inflammatory functions, and then proved to be highly expressed in CECs(Ogata et al., 2002; Becerra, 2006; Zhang et al., 2006). Singh et al. found that CEPCs of mice exposed to dry stress had an amplified immunosuppressive effect on DC maturation, which was eliminated by blocking endogenous PEDF and enhanced by supplementing exogenous recombinant PEDF
(Singh et al., 2020). Their subsequent experiments showed that in vitro culture in the presence of PEDF prevented the reduction in frequency and phenotypic inhibition of regulatory $\mathrm{T}$ cells induced by proinflammatory cytokines (associated with helper $\mathrm{T}$ cells type 17) in normal mice. Their results revealed that PEDF can promote the inhibitory ability of regulatory $\mathrm{T}$ cells and reduce its type 17 helper $\mathrm{T}$ cellmediated dysfunction, thus playing a role in DED inhibition (Singh et al., 2021). Recently, Ma et al. found that PEDF could inhibit the expression of inflammatory cytokines IL-1 $\beta$, IL-6, TNF- $\alpha$, and IL-17A in DED and the percentage of Th17 cells in vivo and in vitro experiments. It was also found that PEDF inhibited the phosphorylation of MAPK p38 and JNK in hypertonic CECs(Ma et al., 2021). All the above studies have shown that PEDF plays anti-inflammatory and immunoregulatory roles in the pathogenesis of DED.

As a myxoid glycoprotein, proteoglycan 4(PRG4) is expressed in the ocular surface, which contributes to the ocular surface integrity and has a good anti-inflammatory effect (Domoto et al., 2002). Menon et al. found that HTCEPI cells synthesized and secreted PRG4, and the secretion of PRG4 was inhibited by TNFa and IL- $1 \beta$ in vitro, and exogenous rhPRG4 could significantly reduce the trend of MIP-1 $\alpha$ and MIP-1 $\beta$ (Menon et al., 2021). Their experiments also found that rhPRG4 can bind to MMP-9 in human tears and inhibit the in vitro activity of exogenous MMP9. In vivo experiments with a mouse DED model showed a significant decrease in PRG4 immunolocalization in corneal epithelium and a significant decrease in the amount of PRG4 in lacrimal gland lysate. These findings may help us to further understand the mechanism of PRG4's immune inflammatory role in the ocular surface.

Thrombocyte reactive protein-1 (TSP-1) is a stromal cell glycoprotein first identified in activated platelets (Lawler, 1978). It can be secreted and expressed in a variety of epithelial cells (Wight et al., 1985). The secretion of TSP-1 is a protective response to inflammation, which can promote the digestion of inflammatory process and accelerate the phagocytosis of damaged cells (Doyen et al., 2003; Grimbert et al., 2006). Tan et al. found that TSP-1 mRNA expression was up-regulated in corneal epithelial cells in DED group. Compared with wild-type mice, the corneal epithelial cells of DED mice were more able to inhibit the expression of MHC-II and CD86 in DC. Moreover, topical application of recombinant TSP-1 significantly inhibited the expression of maturation of DC and proinflammatory cytokine mRNA in the mouse DED model, and improved symptoms. (Tan et al., 2018).

Programmed death ligand 1 (PD-L1) is a member of the receptor $\mathrm{B} 7$ family and plays a role in regulating $\mathrm{T}$-cellmediated immunity (Latchman et al., 2004). Yang et al. found that PD-L1 was highly expressed in the eye cells of DED patients, and it may control inflammation by inhibiting the production of pro-inflammatory cytokines and Th2 cytokines by activated $\mathrm{T}$ cells (Yang et al., 2009). EI Annan et al. found downregulation of corneal epithelial PD-L1 promotes homing of $\mathrm{T}$ cells to the ocular surface by increasing chemokine ligand and receptor expression, thereby amplifying $\mathrm{DED}$ associated keratitis and epithelial lesions. (El Annan et al., 2010). 


\section{Autophagy and Inflammation}

Autophagy is a highly conserved self-degradation process, which has been found in a variety of physiological and pathological processes in the body (Boya et al., 2016; Klionsky et al., 2021). The mechanisms involved in autophagy have been demonstrated in a variety of inflammatory diseases (Lahm and Petrache, 2012; Leung et al., 2017; Lippai and Szatmári, 2017; Rubin et al., 2017; Vij et al., 2018). Autophagy regulates inflammation by affecting the survival, development and homeostasis of inflammatory cells (Saitoh et al., 2008; Zhong et al., 2016; Qian et al., 2017), and affects the transcription, processing and secretion of inflammatory mediators (Crişan et al., 2011). Autophagy is also regulated by inflammatory factors, including IFN- $\gamma$, TNF- $\alpha$, IL- 1, IL-2, IL- 6 , and TGF- $\beta 2$, which can induce autophagy, while IL-4, IL-10, and IL-13 can inhibit autophagy. The role of autophagy in DED is a research hotspot.

Recent studies of DED have shown that autophagy activation can protect the ocular surface from inflammation. Liu Zhao et al. founded that autophagy activation is a late response of HcECs to hyperosmotic stress after inflammation is triggered, which protects HcECs and promotes survival by reducing inflammatory mediators in an vitro model of dry eye. These protective effects were further enhanced when rapamycin enhanced autophagy activation in hypertonic HCECs(Liu et al., 2020a). In addition, they suggest that trehalose, as an autophagy enhancer, induces autophagy antiinflammation by inhibiting Akt activation of transcription factor EB in primary HCECs exposed to high osmotic stress (Liu et al., 2020b). The mechanism of trehalose inhibition of inflammation is independent of NFKB pathway, and it may reduce stress-induced inflammation by inhibiting p38MAPK and activating autophagy (Panigrahi et al., 2019). Therefore, the activation of autophagy is expected to be a new strategy for the treatment of DED.

\section{Pyroptosis and Inflammation}

Pyroptosis is a mechanism of cell death associated with the inflammatory response. Different from cell necrosis, apoptosis and autophagy, pyrodeath can be divided into classical and nonclassical pyrodeath pathways. The classical pathway is induced by caspase-1, while the non-classical pathway relies on caspase- 4 or caspase-5. Pyrotic cells release many inflammatory cytokines, such as IL- $1 \beta$ and IL-18, which trigger the aggregation of immune cells. Pyrolysis is characterized by intact nuclei, DNA strand destruction, and positive TUNEL staining (Guo et al., 2019; Liu and Sun, 2019; Yu et al., 2020) (18-21).

As a member of the immunoglobulin family, triggering receptor expressed on myeloid cells 2 (TREM2) is an immune receptor expressed on the surface of myeloid cells such as microglia, macrophages, osteoclasts and dendritic cells (Colonna, 2003; Daws et al., 2015). TREM2 may exert antiinflammatory effects by enhancing the phagocytosis of myeloid cells (N'Diaye et al., 2009). Qu W. et al. found that compared with wild-type mice, TREM2-deficient mice were more likely to develop keratitis. This is due to the absence of TREM2 leading to increased caspase- 1 and subsequent activation of cell pyroptosis and IL- $1 \beta$ release. In addition, caspase- 1 inhibitors were found to reverse keratopathy in TREM2-deficient mice while inhibiting pyroptosis (Qu et al., 2018).
Pryrin-containing nod-like receptor protein 3(NLRP3) inflammasome is one of the inflammasomes that have been studied extensively. The production of a large number of reactive oxygen species (ROS) can activate NLRP3, and the activation of NLRP3 can activate Caspase-1, which will cause cell Pyroptosis (Mariathasan et al, 2006; Dinarello, 2009) Massive reactive oxygen species (ROS) release is the main characteristic of DED. ROS activate NLRP3 inflammasomes and lead to caspase-1 self-activation and maturation of pro-inflammatory cytokine IL$1 \beta$ in a dry-eye mouse model (Zheng et al., 2014). Niu L et al. found that the mRNA and protein levels of NLRP3 were increased in patients with and without Sjogren's syndrome, and also positively correlated with the severity of dry eye (Niu et al., 2015). The ROS-NLRP3-IL-1 $\beta$ signaling pathway may play an important role in the initiation of environmental induced DE models (Zheng et al., 2015). These findings suggest that NLRP3 inflammasomes may be involved in the development of ocular surface inflammation in DED.

\section{Apoptosis and Inflammation}

Apoptosis is an active, programmed cell death controlled by genes in order to maintain internal environment stability without causing inflammatory response. In dry eyes, the apoptosis of lacrimal acinus, conjunctival epithelium, corneal epithelium, and corneal endothelial cells is abnormally increased, resulting in damage and destruction of eye tissues, while the apoptosis of lymphocytes in local tissues is inhibited, prolonging the survival time of lymphocytes and promoting the inflammatory activation state (Wilson et al., 2002; Yeh et al., 2003; Moore et al., 2011). Cysteine aspartic acid specific protease, p53 protein, and B-cell lymphoma-2 gene (Bcl-2) family proteins are involved in the signal transduction of DED cell apoptosis. Inflammation and apoptosis act together in the pathogenesis of dry eye. In the corneal and conjunctival epithelial cells of diabetic patients, the expression levels of pro-apoptotic factors such as Fas, Fasl and Bax were significantly higher than those of normal subjects, while the expression levels of anti-apoptotic factors such as Bcl-2 were relatively lower. The inflammatory environment of ocular surface can activate pro-apoptotic factors, activate apoptotic signals and activate apoptotic pathways. Moreover, the synergistic effect can further aggravate the apoptosis of corneal epithelial cells, conjunctival epithelial cells and glandular cells, leading to ocular surface abnormalities, and assist in accelerating the occurrence of dry eye disease (Hao et al., 2015). For example, in the occurrence of DED, caspase- 8 and interferon- $\gamma$ (IFN- $\gamma$ ) jointly induce and aggravate the apoptosis of conjunctival cells through the dual apoptotic pathway (X Zhang et al., 2011; X. Zhang, et al., 2014).

\section{LIST THE FACTORS OF DED PATHOGENESIS AND IMMUNE INFLAMMATION}

\section{Sex Hormone and Immune Inflammation}

Epidemiological survey results show that the prevalence of dry eye disease increases significantly with age, especially in women 


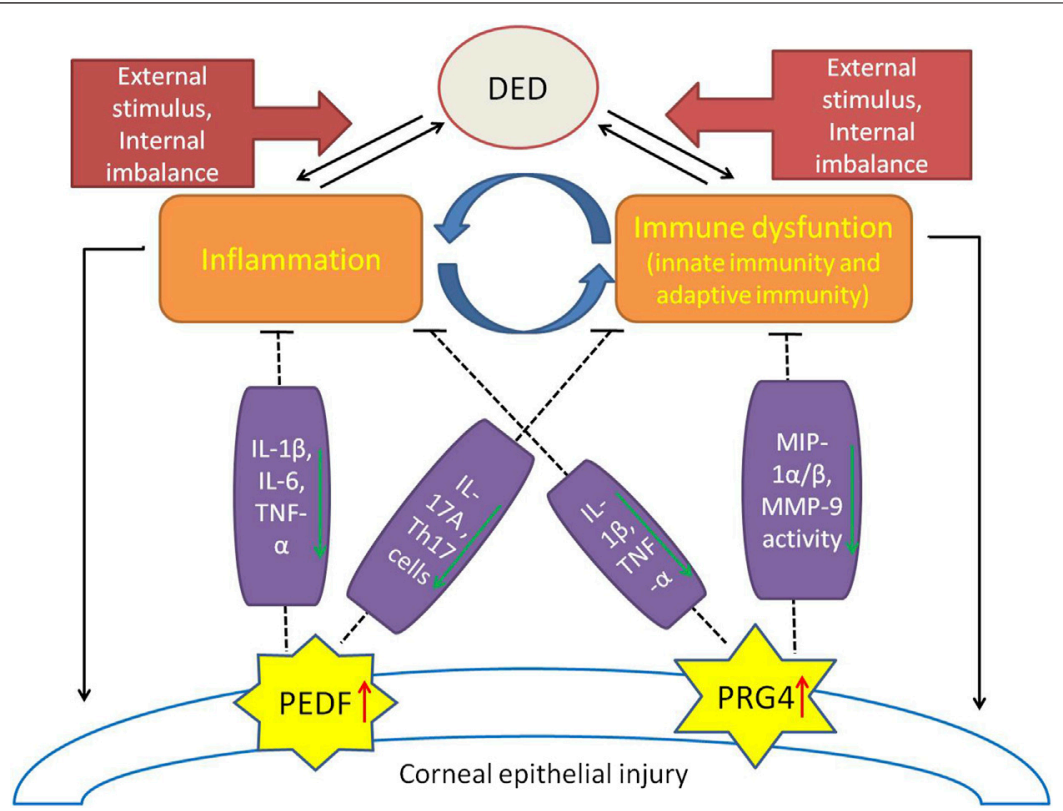

FIGURE 1 |A brief view of the immune-inflammatory mechanisms in DED pathogenesis and the protective effects of immunomodulatory molecules (PEDF, PRG4). External stimulation and internal imbalance lead to the inflammatory initiation of dry eye disease and a vicious cycle of immune regulation dysfunction. Expression of $P E D F$ and PRG4 were up-regulated in damaged corneal endothelial cells. PEDF plays a protective role in DED by inhibiting IL-1 $\beta$, IL-6, TNF- $\alpha$, IL-17A and the percentage of Th17 cells. PRG4 plays an immunomodulatory role in DED by down-regulating IL-1 $\beta$, TNF- $\alpha$, MIP-1 $\alpha / \beta$, and inhibiting the activity of MMP-9.

(Cohen, 2004). Reduced androgen levels may be responsible for the higher prevalence in women than in men.

Androgen plays an important role in the pathogenesis of xerophthalmia and its mechanism is also involved in immune inflammation. Androgens have immunosuppressive effects and can maintain the balance of pro-inflammatory factors and antiinflammatory factors in ocular surface tissues and glands, while the imbalance of androgens will increase the pro-inflammatory factors and cause eye discomfort (Babić et al., 2010; Knop and Knop, 2010; Li and Pflugfelder, 2005). The decrease of androgen level will lead to the atrophy of lacrimal epithelial cells, the disappearance of acinus mucus, the decrease of conjunctival goblet cells, the decrease of mucin expression, the shorten of tear film rupture time, and the decrease of tear quality and quantity (Sullivan et al., 2006). Androgen promotes cholesterol synthesis by regulating the gene expression of the meibomian gland, and the lack of androgen can cause damage to the lipid layer of tear film (Cohen, 2004). Androgen can down-regulate the mRNA expression of small proline rich protein, prevent excessive keratinization of the meibomian gland, and maintain the synthesis and secretion of lipid components in tears from eyelid gland (Sullivan et al., 2009).

Different opinions exist about the role of estrogen in DED and its mechanism. First of all, Zylberberg $\mathrm{C}$ et al. found that estrogen acted on lacrimal glands to increase the secretion of MMP-2 and 9. Suzuki T found that $17-\beta$-estradiol up-regulated the expression of proinflammatory cytokines (IL-1,6,8) and metalloproteinases (MMP-2,7,9) in corneal epithelial cells (Suzuki and Sullivan, 2005). Studies have found that the activation of estrogen receptor $B$ is associated with the down-regulation of the expression of enzymes required for the synthesis of serum lipoxins (LXA4) in corneal epithelial cells (Wang et al., 2011). LXA4 is a negative regulatory signal of some endogenous proinflammatory and pro-proliferation transmitters, which can strongly inhibit the inflammatory response in vivo and inhibit the chemotaxis and adhesion of neutrophils. It can be seen from the above studies that estrogen may promote ocular surface inflammation, and the increase of its level may aggravate ocular surface inflammation, which also explains that estrogen replacement therapy in postmenopausal women can not relieve dry eye symptoms, but may aggravate them. However, in another study, 17- $\beta$-estradiol was found to significantly inhibit IL-1, IL-6, and TNF- $\alpha$ in hypertonic corneal epithelial cells (S. B. Wang, et al., 2011). Ozcura et al. also found that 17- $\beta$-estradiol could inhibit the apoptosis of ocular surface epithelial cells (Ozcura et al., 2012). These also provide some evidence for the treatment of DED with estrogen. Therefore, the role of estrogen in DED needs to be further studied.

\section{Meibomian Gland and Immune Inflammation}

The main function of the meibomian gland is to fight inflammation and infection. Dysfunction of meibomian gland is one of the main causes of DED. When the conjunctival epithelial cells are exposed to bacterial toxins, they can induce significant upregulation of defense genes, expression of cytokines and chemokines, TLR signaling pathway, inflammation and immune response. However, when the epithelial cells of the meibomian gland are exposed to bacterial toxins, they do not 
cause the expression of pro-inflammatory genes and TLR signal transduction. Thus it is speculated that the meibomian gland may have inherent anti-inflammatory and anti-infection factors (Liu et al., 2011). Omiya et al. found that Leucocyte-associated immunoglobulin-like receptor-1 expression is the highest in the meibomian gland, as a kind of inhibitory receptor, could inhibit the activation of immune cells and reduce the production of pro-inflammatory cytokines (Omiya et al., 2009). Furthermore, Leucocyte-associated immunoglobulin-like receptor-1 for differentiation of human meibomian gland epithelial cells was significantly up-regulated (Sullivan et al., 2014). Therefore, we speculate that the meibomian gland may have inherent immune anti-inflammatory and anti-infection mechanisms.

Recent studies have found that a large number of immune inflammatory cells, such as dendritic cells, were detected in corneal epithelial cells and palpebral conjunctival epithelial cells in MGD patients compared with healthy patients (Qazi et al., 2018; Zhou and Robertson, 2018). In addition, there are sex hormone receptors in the palpebral gland and palpebral cells contain enzymes necessary for endocrine synthesis and metabolism of sex hormones and steroids (Knop et al., 2011). Hampel $U$ found that androgens can stimulate eyelid lipid secretion and inhibit inflammation, while estrogen can cause inflammation (Hampel and Garreis, 2017). As a chronic inflammatory disease, MGD induces the infiltration of a large number of immune inflammatory cells, which are important mechanisms leading to the occurrence of dry eye disease.

\section{REFERENCES}

Abehsira-Amar, O., Gibert, M., Joliy, M., Thèze, J., and Jankovic, D. L. (1992). IL-4 Plays a Dominant Role in the Differential Development of Tho into Th1 and Th2 Cells. J. Immunol. 148 (12), 3820-3829.

Ames, P., and Galor, A. (2015). Cyclosporine Ophthalmic Emulsions for the Treatment of Dry Eye: a Review of the Clinical Evidence. Clin. Investig. 5 (3), 267-285. doi:10.4155/cli.14.135

Babić, G. S., Zlatanović, G., Jocić, J. D., Cekić, S., and Vujanović, M. (2010). Therapeutical Approach to Dry Eye Syndrome. Med. Pregl. 63 (11-12), 793-800. doi:10.2298/mpns1012793s

Barbosa, F. L., Pflugfelder, S. C., Coursey, T. G., Bohat, R., and de Paiva, C. S. (2014). Desiccating Stress-Induced Chemokine Expression in the Epithelium Is Dependent on Upregulation of NKG2D/RAE-1 and Release of IFN- $\gamma$ in Experimental Dry Eye. J. Immunol. Official J. Am. Assoc. Immunol. 193, 5264. doi:10.4049/jimmunol.1400016

Baudouin, C. (2001). The Pathology of Dry Eye. Surv. Ophthalmol. 45 (Suppl. 2), S211-S220. doi:10.1016/s0039-6257(00)00200-9

Becerra, S. P. (2006). Focus on Molecules: Pigment Epithelium-Derived Factor (PEDF). Exp. Eye Res. 82 (5), 739-740. doi:10.1016/j.exer.2005.10.016

Boya, P., Esteban-Martínez, L., Serrano-Puebla, A., Gómez-Sintes, R., and Villarejo-Zori, B. (2016). Autophagy in the Eye: Development, Degeneration, and Aging. Prog. Retin. Eye Res. 55, 206-245. doi:10.1016/ j.preteyeres.2016.08.001

Charles, J., Chaperot, L., Salameire, D., Di Domizio, J., and Aspord, C. (2009). Plasmacytoid Dendritic Cells and Dermatological Disorders: Focus on Their Role in Autoimmunity and Cancer. Eur. J. Dermatol. 20 (1), 16-23. doi:10.1684/ ejd.2010.0816

Chauhan, S. K., Annan, J. E., Ecoiffier, T., Goyal, S., Zhang, Q., Saban, D. R., et al. (2009). Autoimmunity in Dry Eye Is Due to Resistance of Th17 to Treg Suppression. J. Immunol. 182 (3), 1247-1252. doi:10.4049/ jimmunol.182.3.1247

\section{CONCLUSION}

As the core focus of the pathogenesis of dry eye disease, immune inflammatory response has always been the focus of scholars and the research direction is relatively extensive. The most recent studies focus on the immunoregulatory molecules expressed by ocular surface cells, especially on PDEF and PRG4 (Figure 1), which have achieved exciting results. The results of these studies can not only provide new targets for the prevention and treatment of DED, but also point out a new direction for our research.

\section{AUTHOR CONTRIBUTION}

LZ and XW initiated the work, designed the idea. YM, RZ, QZ, WL, and WJL prepared and collected material and data. LY, CY, and $\mathrm{HD}$ wrote the paper. All authors reviewed the manuscript. All authors read and approved the final manuscript.

\section{FUNDING}

This work is supported by grants from Liaoning Provincial Natural Science Foundation of China (No.20180550976), Science and Technology Innovation Fund Project of Dalian (No.2019J13SN105), Health Commission Foundation of Dalian (No.1911032).

Chen, Y., Chauhan, S. K., Saban, D. R., Sadrai, Z., Okanobo, A., and Dana, R. (2011). Interferon- $\gamma$-secreting NK Cells Promote Induction of Dry Eye Disease. J. Leukoc. Biol. 89 (6), 965. doi:10.1189/jlb.1110611

Cohen, E. J. (2004). Incidence of Dry Eye in an Older Population. Recent Adv. Ophthalmol. 122 (3), 369. doi:10.1097/00132578-200407000-00007

Colonna, M. (2003). TREMs in the Immune System and beyond. Nat. Rev. Immunol. 3 (6), 445-453. doi:10.1038/nri1106

Contreras-Ruiz, L., and Masli, S. (2015). Immunomodulatory Cross-Talk between Conjunctival Goblet Cells and Dendritic Cells. Plos One 10 (3), e0120284. doi:10.1371/journal.pone.0120284

Corrales, R. M., De Paiva, C. S., Moore, Q. L., Volpe, E. A., Li, D. Q., Gumus, K., et al. (2015). Aqueous Tear Deficiency Increases Conjunctival InterferonGamma (IFN-Gamma) Expression and Goblet Cell Loss. Invest. Ophthalmol. Vis. Sci. 56, 7545. doi:10.1167/iovs.15-17627

Coursey, T. G., Gandhi, N. B., Volpe, E. A., Pflugfelder, S. C., De, P., and Ashour, H. M. (2013). Chemokine Receptors CCR6 and CXCR3 Are Necessary for CD4+ T Cell Mediated Ocular Surface Disease in Experimental Dry Eye Disease. Plos One 8 (11), e78508. doi:10.1371/journal.pone.0078508

Crişan, T., Plantinga, T., van de Veerdonk, F., Farcaş, M., Stoffels, M., Kullberg, B., et al. (2011). Inflammasome-independent Modulation of Cytokine Response by Autophagy in Human Cells. PloS One 6 (4), e18666. doi:10.1371/ journal.pone.0018666

Daws, M. R., Lanier, L. L., Seaman, W. E., and Ryan, J. C. (2015). Cloning and Characterization of a Novel Mouse Myeloid DAP12-Associated Receptor Family. Eur. J. Immunol. 31 (3), 783-791. doi:10.1002/1521-4141(200103)31: 3<783::aid-immu783>3.0.co;2-u

Dinarello, C. A. (2009). Immunological and Inflammatory Functions of the Interleukin-1 Family. Annu. Rev. Immunol. 27 (1), 519-550. doi:10.1146/ annurev.immunol.021908.132612

Dohlman, T. H., Chauhan, S. K., Kodati, S., Hua, J., Chen, Y., Omoto, M., et al. (2013). The CCR6/CCL20 Axis Mediates Th17 Cell Migration to the Ocular Surface in Dry Eye Disease. Invest. Ophthalmol. Vis. Sci. 54 (6), 4081-4091. doi:10.1167/iovs.12-11216 
Domoto, H., Hosaka, T., Oikawa, K., Ohbayashi, T., Ishida, T., Izumi, M., et al. (2002). TLS-CHOP Target Gene DOL54 Expression in Liposarcomas and Malignant Fibrous Histiocytomas. Pathol. Int. 52 (8), 497-500. doi:10.1046/ j.1440-1827.2002.01391.x

Doyen, V., Rubio, M., Braun, D., Nakajima, T., Abe, J., Saito, H., et al. (2003). Thrombospondin 1 Is an Autocrine Negative Regulator of Human Dendritic Cell Activation. J. Exp. Med. 198 (8), 1277-1283. doi:10.1084/jem.20030705

Du, S., Hiramatsu, N., Hayakawa, K., Kasai, A., and Kitamura, M. (2009). Suppression of NF- B by Cyclosporin A and Tacrolimus (FK506) via Induction of the C/EBP Family: Implication for Unfolded Protein Response. J. Immunol. 182 (11), 7201-7211. doi:10.4049/jimmunol.0801772

Duque, G. A., and Descoteaux, A. (2014). Macrophage Cytokines: Involvement in Immunity and Infectious Diseases. Front. Immunol. 5 (491), 491. doi:10.3389/ fimmu.2014.00491

El Annan, J., Goyal, S., Zhang, Q., Freeman, G., Sharpe, A., and Dana, R. (2010). Regulation of T-Cell Chemotaxis by Programmed Death-Ligand 1 (PD-L1) in Dry Eye-Associated Corneal Inflammation. Invest. Ophthalmol. Vis. Sci. 51 (7), 3418-3423. doi:10.1167/iovs.09-3684

Enríquez-de-Salamanca, A., Castellanos, E., Stern, M., Fernández, I., Carreño, E., García-Vázquez, C., et al. (2010). Tear Cytokine and Chemokine Analysis and Clinical Correlations in Evaporative-type Dry Eye Disease. Mol. Vis. 16, 862-873.

Farrand, K., Fridman, M., Stillman, I., and Schaumberg, D. (2017). Prevalence of Diagnosed Dry Eye Disease in the United States Among Adults Aged 18 Years and Older. Am. J. Ophthalmol. 182, 90-98. doi:10.1016/j.ajo.2017.06.033

Gollmer, K., Asperti-Boursin, F., Tanaka, Y., Okkenhaug, K., and Stein, J. V. (2009). CCL21 Mediates CD4+ T-Cell Costimulation via a DOCK2/Rac-dependent Pathway. Blood 114 (3), 580-588. doi:10.1182/blood-2009-01-200923

Grimbert, P., Bouguermouh, S., Baba, N., Nakajima, T., Allakhverdi, Z., Braun, D., et al. (2006). Thrombospondin/CD47 Interaction: a Pathway to Generate Regulatory $\mathrm{T}$ Cells from Human CD4+ CD25- T Cells in Response to Inflammation. J. Immunol. 177 (6), 3534-3541. doi:10.4049/ jimmunol.177.6.3534

Guo, B., Lu, P., Chen, X., Zhang, W., and Chen, R. (2010). Prevalence of Dry Eye Disease in Mongolians at High Altitude in China: the Henan Eye Study. Ophthal. Epidemiol. 17 (4), 234-241. doi:10.3109/09286586.2010.498659

Guo, H., Xie, M., Zhou, C., and Zheng, M. (2019). The Relevance of Pyroptosis in the Pathogenesis of Liver Diseases. Life Sci. 223, 69. doi:10.1016/ j.lfs. 2019.02 .060

Hackstein, H., Tschipakow, I., Bein, G., Nold, P., Brendel, C., and Baal, N. (2016). Contact-dependent Abrogation of Bone Marrow-Derived Plasmacytoid Dendritic Cell Differentiation by Murine Mesenchymal Stem Cells. Biochem. Biophys. Res. Commun. 476 (1), 15-20. doi:10.1016/j.bbrc.2016.05.108

Hampel, U., and Garreis, F. (2017). The Human Meibomian Gland Epithelial Cell Line as a Model to Study Meibomian Gland Dysfunction. Exp. Eye Res. 163, 46-52. doi:10.1016/j.exer.2017.03.011

Hao, L., Sheng, M., Yu, L., Peng, W., and Bing, L. (2015). Expression of SIRT1 and Oxidative Stress in Diabetic Dry Eye. Int. J. Clin. Exp. Pathol. 8 (6), 7644.

Hui, L., Jing, Z., Kumar, A., Mei, Z., Atherton, S. S., and Yu, F. (2010). Herpes Simplex Virus 1 Infection Induces the Expression of Proinflammatory Cytokines, Interferons and TLR7 in Human Corneal Epithelial Cells. Insect Sci. 117 (2), 167-176. doi:10.1111/j.1365-2567.2005.02275.x

Jaafar, E. A., Chauhan, S. K., Tatiana, E., Zhang, Q., Saban, D. R., and Reza, D. (2009). Characterization of Effector $\mathrm{T}$ Cells in Dry Eye Disease. Invest. Ophthalmol. Vis. Sci. 50 (8), 3802.

Jego, G., Palucka, A. K., Blanck, J. P., Chalouni, C., Pascual, V., and Banchereau, J. (2003). Plasmacytoid Dendritic Cells Induce Plasma Cell Differentiation through Type I Interferon and Interleukin 6. Immunity 19 (2), 225-234. doi:10.1016/s1074-7613(03)00208-5

Ji, Y. W., Seo, Y., Choi, W., Yeo, A., Noh, H., Kim, E. K., et al. (2014). Dry EyeInduced CCR7+CD11b+ Cell Lymph Node Homing Is Induced by COX-2 Activities. Invest. Ophthalmol. Vis. 55 (10), 6829-6838. doi:10.1167/iovs.1414744

Jing, L., Xu, R., Hu, L. T., Jia, Y., Jiang, N., Cui, L., et al. (2018). Interleukin-32 Induced Thymic Stromal Lymphopoietin Plays a Critical Role in the Inflammatory Response in Human Corneal Epithelium. Cell Signal. 49, 39. doi:10.1016/j.cellsig.2018.05.007
Klionsky, D., Abdel-Aziz, A., Abdelfatah, S., Abdellatif, M., Abdoli, A., Abel, S., et al. (2021). Guidelines for the Use and Interpretation of Assays for Monitoring Autophagy (4th Edition). Autophagy 17 (1), 1-382. doi:10.1080/ 15548627.2020 .1797280

Knop, E., Knop, N., Millar, T., Obata, H., and Sullivan, D. (2011). The International Workshop on Meibomian Gland Dysfunction: Report of the Subcommittee on Anatomy, Physiology, and Pathophysiology of the Meibomian Gland. Invest. Ophthalmol. Vis. Sci. 52 (4), 1938-1978. doi:10.1167/iovs.10-6997c

Knop, N., and Knop, E. (2010). Regulation of the Inflammatory Component in Chronic Dry Eye Disease by the Eye-Associated Lymphoid Tissue (EALT). Dev. Ophthalmol. 45, 23-39. doi:10.1159/000315017

Kodati, S., Chauhan, S. K., Chen, Y., Dohlman, T. H., Karimian, P., Saban, D., et al. (2014). CCR7 Is Critical for the Induction and Maintenance of Th17 Immunity in Dry Eye Disease. Invest. Ophthalmol. Vis. 55 (9), 5871-5877. doi:10.1167/ iovs.14-14481

Lahm, T., and Petrache, I. (2012). LC3 as a Potential Therapeutic Target in Hypoxia-Induced Pulmonary Hypertension. Autophagy 8 (7), 1146-1147. doi:10.4161/auto.20520

Lam, H., Bleiden, L., de Paiva, C., Farley, W., Stern, M., and Pflugfelder, S. (2009). Tear Cytokine Profiles in Dysfunctional Tear Syndrome. Am. J. Ophthalmol. 147 (2), 198-205. doi:10.1016/j.ajo.2008.08.032

Latchman, Y. E., Liang, S. C., Yin, W., Chernova, T., and Sharpe, A. H. (2004). PDL1-deficient Mice Show that PD-L1 on T Cells, Antigen-Presenting Cells, and Host Tissues Negatively Regulates T Cells. Proc. Natl. Acad. Sci. 101 (29), 10691-10696. doi:10.1073/pnas.0307252101

Lawler, J. (1978). Isolation and Characterization of a High Molecular Weight Glycoprotein from Human Blood Platelets. J. Biol. Chem. 253 (23), 8609-8616. doi:10.1016/s0021-9258(17)34336-3

Lee, H. S., Hattori, T., Park, E. Y., Stevenson, W., Chauhan, S. K., and Dana, R. (2012). Expression of Toll-Like Receptor 4 Contributes to Corneal Inflammation in Experimental Dry Eye Disease. Invest. Ophthalmol. Vis. 53 (9), 5632-5640. doi:10.1167/iovs.12-9547

Leung, G., Ying, D., Mak, C., Chen, X., Xu, W., Yeung, K., et al. (2017). CFTR Founder Mutation Causes Protein Trafficking Defects in Chinese Patients with Cystic Fibrosis. Mol. Genet. Genomic Med. 5 (1), 40-49. doi:10.1002/mgg3.258

Li, D. Q., and Pflugfelder, S. C. (2005). Matrix Metalloproteinases in Corneal Inflammation. Ocul. Surf. 3 (4), S198. doi:10.1016/s1542-0124(12)70255-0

Li, D. Q., Zhuo, C., Xiu, J. S., Luo, L., and Pflugfelder, S. C. (2013). Stimulation of Matrix Metalloproteinases by Hyperosmolarity via a JNK Pathway in Human Corneal Epithelial Cells. Invest. Ophthalmol. Vis. Sci. 45 (12), 4302.

Lippai, M., and Szatmári, Z. (2017). Autophagy-from Molecular Mechanisms to Clinical Relevance. Cel Biol. Toxicol. 33 (2), 145-168. doi:10.1007/s10565-0169374-5

Liu, L., and Sun, B. (2019). Neutrophil Pyroptosis: New Perspectives on Sepsis. Cell Mol. Life Sci. 76, 2031. doi:10.1007/s00018-019-03060-1

Liu, S., Richards, S. M., Kristine, L., Mark, H., Aaron, F., and Sullivan, D. A. (2011). Changes in Gene Expression in Human Meibomian Gland Dysfunction. Invest. Ophthalmol. Vis. Sci. 52 (5), 2727-2740. doi:10.1167/iovs.10-6482

Liu, Z., Chen, D., Chen, X., Bian, F., and Li, D. Q. (2020a). Autophagy Activation Protects Ocular Surface from Inflammation in a Dry Eye Model In Vitro. Int. J. Mol. Sci. 21 (23), 8966. doi:10.3390/ijms 21238966

Liu, Z., Chen, D., Chen, X., Bian, F., and Li, D. Q. (2020b). Trehalose Induces Autophagy against Inflammation by Activating TFEB Signaling Pathway in Human Corneal Epithelial Cells Exposed to Hyperosmotic Stress. Invest. Ophthalmol. Vis. Sci. 61 (10), 26. doi:10.1167/iovs.61.10.26

Ma, B., Zhou, Y., Liu, R., Zhang, K., Yang, T., Hu, C., et al. (2021). Pigment Epithelium-Derived Factor (PEDF) Plays Anti-inflammatory Roles in the Pathogenesis of Dry Eye Disease. Ocul. Surf. 20, 70-85. doi:10.1016/ j.jtos.2020.12.007

Mariathasan, S., Weiss, D. S., Newton, K., Mcbride, J., O'Rourke, K., Roose-Girma, M., et al. (2006). Cryopyrin Activates the Inflammasome in Response to Toxins and ATP. Nature 440, 228.

Marko, C. K., Menon, B. B., Chen, G., Whitsett, J. A., Clevers, H., and Gipson, I. K. (2013). Spdef Null Mice Lack Conjunctival Goblet Cells and Provide a Model of Dry Eye. Am. J. Pathol. 183 (1), 35-48. doi:10.1016/j.ajpath.2013.03.017

Marsland, B. J., BäTtig, P., Bauer, M., Ruedl, C., LiSsing, U., Beerli, R. R., et al. (2005). CCL19 and CCL21 Induce a Potent Proinflammatory Differentiation 
Program in Licensed Dendritic Cells. Immunity 22 (4), 493-505. doi:10.1016/ j.immuni.2005.02.010

Matsuda, S., and Koyasu, S. (2000). Mechanisms of Action of Cyclosporine. Immunopharmacology 47, 119. doi:10.1016/s0162-3109(00)00192-2

Matsuda, S., Moriguchi, T., Koyasu, S., and Nishida, E. (1998). T Lymphocyte Activation Signals for Interleukin-2 Production Involve Activation of MKK6P38 and MKK7-SAPK/JNK Signaling Pathways Sensitive to Cyclosporin A. J. Biol. Chem. 273 (20), 12378-12382. doi:10.1074/jbc.273.20.12378

Menon, N., Goyal, R., Lema, C., Woods, P., Tanguay, A., Morin, A., et al. (2021). Proteoglycan 4 (PRG4) Expression and Function in Dry Eye Associated Inflammation. Exp. Eye Res. 208, 108628. doi:10.1016/j.exer.2021.108628

Mesraoua, R., Falcone, D. J., Khan, K. M., Kothari, P., Pestana, R., and Elchaki, R. (2014). IL-6-mediated Induction of Matrix Metalloproteinase-9 Is Modulated by JAK-dependent IL-10 Expression in Macrophages. J. Immunol. 192, 349. doi:10.4049/jimmunol.1301906

Milner, M. S., Beckman, K. A., Luchs, J. I., Allen, Q. B., and Yeu, E. (2017). Dysfunctional Tear Syndrome: Dry Eye Disease and Associated Tear Film Disorders - New Strategies for Diagnosis and Treatment. Curr. Opin. Ophthalmol. 27, 3. doi:10.1097/01.icu.0000512373.81749.b7

Moore, J. E., Vasey, G. T., Dartt, D. A., Mcgilligan, V. E., Atkinson, S. D., Grills, C., et al. (2011). Effect of Tear Hyperosmolarity and Signs of Clinical Ocular Surface Pathology upon Conjunctival Goblet Cell Function in the Human Ocular Surface. Invest. Ophthalmol. Vis. 52 (9), 6174-6180. doi:10.1167/ iovs.10-7022

N'Diaye, E-N., Branda, C. S., Branda, S. S., Nevarez, L., Colonna, M., Lowell, C., et al. (2009). TREM-2 (Triggering Receptor Expressed on Myeloid Cells 2) Is a Phagocytic Receptor for Bacteria. J. Cel Biol. 184, 215. doi:10.1083/ jcb. 200808080

Nelson, J. D., Craig, J. P., Akpek, E. K., Azar, D. T., and Sullivan, D. A. (2017). TFOS DEWS II Introduction. Ocul. Surf. 15 (3), 276. doi:10.1016/j.jtos.2017.05.005

Niu, L., Zhang, S., Wu, J., Ling, C., Wang, Y., and Fulvio, D. (2015). Upregulation of NLRP3 Inflammasome in the Tears and Ocular Surface of Dry Eye Patients. Plos One 10 (5), e0126277. doi:10.1371/journal.pone.0126277

Ogata, N., Wada, M., Otsuji, T., Jo, N., and Matsumura, M. (2002). Expression of Pigment Epithelium-Derived Factor in normal Adult Rat Eye and Experimental Choroidal Neovascularization. Invest. Ophthalmol. Vis. 43 (4), 1168.

Omiya, R., Tsushima, F., Narazaki, H., Sakoda, Y., Kim, Y., and Kuramasu, A. (2009). Leucocyte-associated Immunoglobulin-like Receptor-1 Is an Inhibitory Regulator of Contact Hypersensitivity. Immunology 128, 543. doi:10.1111/ j.1365-2567.2009.03140.x

Ozcura, F., Dündar, S. O., Cetin, E. D., Beder, N., and Dündar, M. (2012). Effects of Estrogen Replacement Therapy on Apoptosis and Vascular Endothelial Growth Factor Expression in Ocular Surface Epithelial cells:An Experimental Study. Int. J. Ophthalmol. 5 (001), 64-68. doi:10.3980/j.issn.2222-3959.2012.01.13

Pan, Z. J., Horton, C. G., Lawrence, C., and Farris, A. D. (2016). Plasmacytoid Dendritic Cells and Type 1 Interferon Promote Peripheral Expansion of Forkhead Box Protein 3+ Regulatory T Cells Specific for the Ubiquitous RNA-binding Nuclear Antigen La/Sjgren's Syndrome (SS)-B. Clin. Exp. Immunol. 186 (1), 18-29. doi:10.1111/cei.12817

Panigrahi, T., Shivakumar, S., Shetty, R., D'souza, S., Nelson, E., Sethu, S., et al. (2019). Trehalose Augments Autophagy to Mitigate Stress Induced Inflammation in Human Corneal Cells. Ocul. Surf. 17 (4), 699-713. doi:10.1016/j.jtos.2019.08.004

Perez, V. L., Pflugfelder, S. C., Zhang, S., Shojaei, A., and Haque, R. (2016). Lifitegrast, a Novel Integrin Antagonist for Treatment of Dry Eye Disease. Ocul. Surf. 14 (2), 207-215. doi:10.1016/j.jtos.2016.01.001

Periman, L. M., Perez, V. L., Saban, D. R., Lin, M. C., and Neri, P. (2020). The Immunological Basis of Dry Eye Disease and Current Topical Treatment Options. J. Ocul. Pharmacol. Ther. 36 (3), 137. doi:10.1089/jop.2019.0060

Pflugfelder, S. C., and Paiva, C. (2017). The Pathophysiology of Dry Eye Disease: What We Know and Future Directions for Research. Ophthalmology 124 (11), S4-S13. doi:10.1016/j.ophtha.2017.07.010

Pierre, N., Hong, L., Elodie, R., Ghislaine, R., Elise, W., Francoise, B., et al. (2018). Proinflammatory Markers, Chemokines, and Enkephalin in Patients Suffering from Dry Eye Disease. Int. J. Mol. Sci. 19 (4), 1221.

Qazi, Y., Kheirkhah, A., Blackie, C., Trinidad, M., Williams, C., Cruzat, A., et al. (2018). Clinically Relevant Immune-Cellular Metrics of Inflammation in
Meibomian Gland Dysfunction. Invest. Opthalmol. Vis. Sci. 59 (15), 6111. doi:10.1167/iovs.18-25571

Qian, M., Fang, X., and Wang, X. (2017). Autophagy and Inflammation. Clin. Translational Med. 6 (1), 24. doi:10.1186/s40169-017-0154-5

Qu, W., Wang, Y., Wu, Y., Liu, Y., Chen, K., Liu, X., et al. (2018). Triggering Receptors Expressed on Myeloid Cells 2 Promotes Corneal Resistance against Pseudomonas aeruginosa by Inhibiting Caspase-1-Dependent Pyroptosis. Front. Immunol. 9, 1121. doi:10.3389/fimmu.2018.01121

Rubin, D., Feld, L., Goeppinger, S., Margolese, J., Rosh, J., Rubin, M., et al. (2017). The Crohn's and Colitis Foundation of America Survey of Inflammatory Bowel Disease Patient Health Care Access. Inflamm. Bowel Dis. 23 (2), 224-232. doi:10.1097/mib.0000000000000994

Saitoh, T., Fujita, N., Jang, M., Uematsu, S., Yang, B., Satoh, T., et al. (2008). Loss of the Autophagy Protein Atg16L1 Enhances Endotoxin-Induced IL-1beta Production. Nature 456 (7219), 264-268. doi:10.1038/nature07383

Schaumburg, C. S., Siemasko, K. F., Paiva, C. D., Wheeler, L. A., Niederkorn, J. Y., Pflugfelder, S. C., et al. (2011). Ocular Surface APCs Are Necessary for Autoreactive $\mathrm{T}$ Cell-Mediated Experimental Autoimmune Lacrimal Keratoconjunctivitis. J. Immunol. 187 (7), 3653-3662. doi:10.4049/ jimmunol.1101442

Seung, P. B., Yeon, C. Y., Adhikari, N., Neupane, S., Kim, H., Lee, D. C., et al. (2019). Cyclosporine A Eyedrops with Self-Nanoemulsifying Drug Delivery Systems Have Improved Physicochemical Properties and Efficacy against Dry Eye Disease in a Murine Dry Eye Model. PloS one 14 (11), e0224805.

Singh, R. B., Blanco, T., Mittal, S. K., Alemi, H., and Dana, R. (2021). Pigment Epithelium-Derived Factor Enhances the Suppressive Phenotype of Regulatory T Cells in a Murine Model of Dry Eye Disease. Am. J. Pathol. 191, 720. doi:10.1016/j.ajpath.2021.01.003

Singh, R. B., Blanco, T., Mittal, S. K., Taketani, Y., and Dana, R. (2020). Pigment Epithelium-Derived Factor Secreted by Corneal Epithelial Cells Regulates Dendritic Cell Maturation in Dry Eye Disease. Ocul. Surf. 18 (3), 460. doi:10.1016/j.jtos.2020.05.002

Stapleton, F., Alves, M., Bunya, V., Jalbert, I., Lekhanont, K., Malet, F., et al. (2017). TFOS DEWS II Epidemiology Report. Ocul. Surf. 15 (3), 334-365. doi:10.1016/ j.jtos.2017.05.003

Stern, M. E., Schaumburg, C. S., Dana, R., Calonge, M., Niederkorn, J. Y., and Pflugfelder, S. C. (2010). Autoimmunity at the Ocular Surface: Pathogenesis and Regulation. Mucosal Immunol. 3 (5), 425. doi:10.1038/mi.2010.26

Stern, M. E., Schaumburg, C. S., and Pflugfelder, S. C. (2013). Dry Eye as a Mucosal Autoimmune Disease. Int. Rev. Immunol. 32 (1), 19-41. doi:10.3109/ 08830185.2012.748052

Sullivan, D. A., Jensen, R. V., Suzuki, T., and Richards, S. M. (2009). Do sex Steroids Exert Sex-specific And/or Opposite Effects on Gene Expression in Lacrimal and Meibomian Glands?. Mol. Vis. 15 (166), 1553.

Sullivan, D. A., Liu, Y., Kam, W. R., Ding, J., Green, K. M., Shaffer, S. A., et al. (2014). Serum-Induced Differentiation of Human Meibomian Gland Epithelial Cells. Invest. Ophthalmol. Vis. Sci. 55 (6), 3866. doi:10.1167/iovs.13-13407

Sullivan, D. A., Wickham, L. A., Rocha, E. M., Krenzer, K. L., and Toda, I. (2006). Androgens and Dry Eye in Sjgren's Syndrome. Ann. N Y Acad. Sci. 876 (1), 312-324. doi:10.1111/j.1749-6632.1999.tb07656.x

Surendar, J., Mohan, V., Pavankumar, N., Babu, S., and Aravindhan, V. (2011). Increased Levels of Serum Granulocyte-Macrophage colony-stimulating Factor Is Associated with Activated Peripheral Dendritic Cells in Type 2 Diabetes Subjects (CURES-99). Diabetes Tech. Ther. 14 (4), 344-349. doi:10.1089/ dia.2011.0182

Suzuki, T., and Sullivan, D. A. (2005). Estrogen Stimulation of Proinflammatory Cytokine and Matrix Metalloproteinase Gene Expression in Human Corneal Epithelial Cells. Cornea 24 (8), 1004-1009. doi:10.1097/ 01.ico.0000160973.04072.a5

Tan, X., Chen, Y., Foulsham, W., Amouzegar, A., Inomata, T., Liu, Y., et al. (2018). The Immunoregulatory Role of Corneal Epithelium-Derived Thrombospondin-1 in Dry Eye Disease. Ocul. Surf. 16 (4), 470-477. doi:10.1016/j.jtos.2018.07.005

Vij, N., Chandramani-Shivalingappa, P., Van Westphal, C., Hole, R., and Bodas, M. (2018). Cigarette Smoke-Induced Autophagy Impairment Accelerates Lung Aging, COPD-Emphysema Exacerbations and Pathogenesis. Am. J. Physiol. Cel Physiol. 314 (1), C73-C87. doi:10.1152/ajpcell.00110.2016 
Wan, K. H., Chen, L. J., and Young, A. L. (2015). Efficacy and Safety of Topical $0.05 \%$ Cyclosporine Eye Drops in the Treatment of Dry Eye Syndrome: A Systematic Review and Meta-Analysis. Ocul. Surf. 13, 213. doi:10.1016/ j.jtos.2014.12.006

Wang, S. B., Hu, K. M., Seamon, K. J., Mani, V., and Gronert, K. (2011). Estrogen Negatively Regulates Epithelial Wound Healing and Protective Lipid Mediator Circuits in the Cornea. FASEB J. 26 (4), 1506-1516. doi:10.1096/fj.11-198036

Wang, S., and Zhang, H. (2019). Upregulation of the IL-33/ST2 Pathway in Dry Eye. Mol. Vis. 25, 583-592.

Wight, T. N., Raugi, G. J., Mumby, S. M., and Bornstein, P. (1985). Light Microscopic Immunolocation of Thrombospondin in Human Tissues. J. Histochem. Cytochem. Official J. Histochem. Soc. 33 (4), 295-302. doi:10.1177/33.4.3884704

Wilson, S., Mohan, R., Hong, J., Lee, J., Choi, R., Liu, J., et al. (2002). Apoptosis in the Cornea in Response to Epithelial Injury: Significance to Wound Healing and Dry Eye. Adv. Exp. Med. Biol. 506, 821-826. doi:10.1007/978-1-4615-0717$8 \_116$

Yang, W., Li, H., Chen, P., Alizadeh, H., He, Y., Hogan, R., et al. (2009). PD-L1 Expression on Human Ocular Cells and its Possible Role in Regulating Immune-Mediated Ocular Inflammation. Invest. Ophthalmol. Vis. Sci. 50 (1), 273-280. doi:10.1167/iovs.08-2397

Yeh, S., Song, X. J., Farley, W., Li, D. Q., Stern, M. E., and Pflugfelder, S. C. (2003). Apoptosis of Ocular Surface Cells in Experimentally Induced Dry Eye. Invest. Ophthalmol. Vis. 44 (1), 124. doi:10.1167/iovs.02-0581

You, J., Lin, J., Zhou, Y., Peng, X., He, H., Li, C., et al. (2019). Aspergillus fumigatusRole of the IL-33/ST2/p38 Signaling Pathway in the Immune Response of Corneal Epithelial Cells to Infection. Int. J. Ophthalmol. 12 (4), 549-556. doi:10.18240/ijo.2019.04.04

Yu, Z., Zhang, J., Li, X., Wang, Y., Fu, Y., and Gao, X. (2020). A New Research Hot Spot: The Role of NLRP3 Inflammasome Activation, a Key Step in Pyroptosis, in Diabetes and Diabetic Complications. Life Sci. 240, 117138. doi:10.1016/ j.lfs.2019.117138

Zhang, S. X., Wang, J. J., Guoquan, G., Chunkui, S., Robert, M., and Jian-Xing, M. (2006). Pigment Epithelium-Derived Factor (PEDF) is an Endogenous Antiinflammatory Factor. Faseb J. 20 (2), 323-325. doi:10.1096/fj.05-4313fje

Zhang, X., Chen, W., De Paiva, C., Corrales, R., Volpe, E., McClellan, A., et al. (2011). Interferon- $\gamma$ Exacerbates Dry Eye-Induced Apoptosis in Conjunctiva through Dual Apoptotic Pathways. Invest. Ophthalmol. Vis. Sci. 52 (9), 6279-6285. doi:10.1167/iovs.10-7081

Zhang, X., Paiva, C. D., Su, Z., Volpe, E. A., Li, D. Q., and Pflugfelder, S. C. (2014). Topical Interferon-Gamma Neutralization Prevents Conjunctival Goblet Cell
Loss in Experimental Murine Dry Eye. Exp. Eye Res. 118, 117-124. doi:10.1016/ j.exer.2013.11.011

Zhang, X., Volpe, E., Gandhi, N. B., Schaumburg, C. S., Siemasko, K. F., Pangelinan, S. B., et al. (2012). NK Cells Promote Th-17 Mediated Corneal Barrier Disruption in Dry Eye. Plos One 7 (5), e36822. doi:10.1371/ journal.pone.0036822

Zheng, Q., Ren, Y., Reinach, P. S., She, Y., Xiao, B., Hua, S., et al. (2014). Reactive Oxygen Species Activated NLRP3 Inflammasomes Prime EnvironmentInduced Murine Dry Eye. Exp. Eye Res. 125, 1-8. doi:10.1016/j.exer.2014.05.001

Zheng, Q., Ren, Y., Reinach, P. S., Xiao, B., Lu, H., Zhu, Y., et al. (2015). Reactive Oxygen Species Activated NLRP3 Inflammasomes Initiate Inflammation in Hyperosmolarity Stressed Human Corneal Epithelial Cells and EnvironmentInduced Dry Eye Patients. Exp. Eye Res. 134, 133-140. doi:10.1016/ j.exer.2015.02.013

Zhong, Z., Sanchez-Lopez, E., and Karin, M. (2016). Autophagy, Inflammation, and Immunity: A Troika Governing Cancer and its Treatment. Cell 166 (2), 288-298. doi:10.1016/j.cell.2016.05.051

Zhou, S., and Robertson, D. (2018). Wide-Field In Vivo Confocal Microscopy of Meibomian Gland Acini and Rete Ridges in the Eyelid Margin. Invest. Ophthalmol. Vis. Sci. 59 (10), 4249-4257. doi:10.1167/iovs.18-24497

Conflict of Interest: The authors declare that the research was conducted in the absence of any commercial or financial relationships that could be construed as a potential conflict of interest.

The handling editor declared a shared affiliation with several of the authors LY, CY, HD, RZ, QZ, WL, WL, XW, and LZ at time of review.

Publisher's Note: All claims expressed in this article are solely those of the authors and do not necessarily represent those of their affiliated organizations, or those of the publisher, the editors and the reviewers. Any product that may be evaluated in this article, or claim that may be made by its manufacturer, is not guaranteed or endorsed by the publisher.

Copyright (c) $2021 \mathrm{Yu}$, Yu, Dong, Mu, Zhang, Zhang, Liang, Li, Wang and Zhang. This is an open-access article distributed under the terms of the Creative Commons Attribution License (CC BY). The use, distribution or reproduction in other forums is permitted, provided the original author(s) and the copyright owner(s) are credited and that the original publication in this journal is cited, in accordance with accepted academic practice. No use, distribution or reproduction is permitted which does not comply with these terms. 